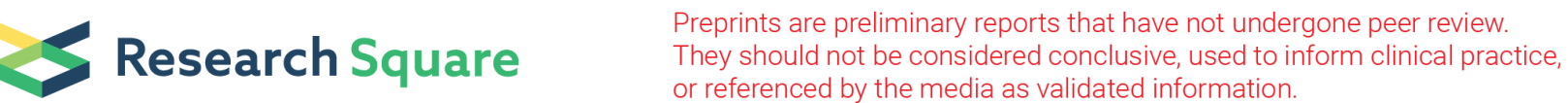

\section{Comparison of various aspects of women's lives between infertile and women with and without tubal ligation: a comparative cross-sectional study}

Mahnaz Ashrafi

Royan Institute

Shahideh Jahanian Sadatmahalleh ( $\sim$ shahideh.jahanian@modares.ac.ir)

Tarbiat Modares University Faculty of Medical Sciences https://orcid.org/0000-0002-7006-8487

Negin Mirzaei

Tarbiat Modares University Faculty of Medical Sciences

Nadia Jahangiri,

Royan Institute

Samaneh Youseflu

Zanjan University of Medical Sciences

Malihe Nasiri

Shahid Beheshti University of Medical Sciences

Research article

Keywords: Infertility, Tubal Ligation, Sexual Function, Quality of Life, Depression

Posted Date: January 11th, 2021

DOI: https://doi.org/10.21203/rs.3.rs-38747/v2

License: (c) (1) This work is licensed under a Creative Commons Attribution 4.0 International License.

Read Full License 


\section{Abstract}

Background: The aim of this study is to compare anxiety, depression, body image, self-esteem, sexual function (SF), and quality of life (QoL) between infertile women and women with or without tubal ligation $(\mathrm{TL})$.

Methods: This cross-sectional study was conducted on 600 women, distributed equally as infertile women, women with or without TL, who met the inclusion criteria. They were selected from the Royan Institute in Tehran (Iran) and a number of health care centers in Tehran (Iran) from May 2017 to February 2019. The subjects were asked to fill out the Short Form Health Survey (SF-12), Female Sexual Function Index (FSFI), Hospital Anxiety and Depression Scale (HADS), Body Image Concern Inventory (BICl), and Rosenberg' Self-Esteem Scale (RSES). One-way ANOVA was used to find the statistical differences between the three groups.

Results: Mean scores of all FSFI domains were found to be lower in the TL women and the differences between the three groups were statistically significant in all domains. Women with TL had more female sexual dysfunction (FSD) (22.43 \pm 5.30 vs $24.79 \pm 4.74$ vs $28.03 \pm 3.29$, P $\llbracket 0.001)$. There was a significant difference between the three groups in SF-12 scores ( $76.59 \pm 13.14$ vs $68.49 \pm 14.47$ vs $78.87 \pm 12.62$, $P \llbracket 0.001)$. Also, there was a significant difference between the three groups in anxiety, depression, and total scores HADS (Pख0.001). Infertile women had lower body image (Pख0.05) and self-esteem was lower in the TL group (Pख0.05).

Conclusions: Our findings reveal the adverse effects of TL on the anxiety and depression, sexual life, body image, and QoL of women. It is recommended that the awareness and knowledge of health-care professionals regarding the side-effects of TL in the above-mentioned aspects of women's lives should be increased and discussed with patients.

\section{Background}

Childbearing is one of the main goals of family formation, which has a special socio-cultural and religious significance in Iranian culture. As a matter of fact, it is an important expected outcome of their sexual relationship for most couples, and many factors that may disrupt the natural fertility process impose an enormous psychological burden on a couples' life $(1,2)$.

Infertility is the inability to conceive after 12 months of frequent coitus without contraceptives, which accounts for 25 percent of couples in developing countries (3). Infertility imposes a great deal of psychological burden to couples and has negative effects on various aspects of their lives(4) and can increase marital conflicts and sexual dysfunction (5). Anecdotal evidence suggests that infertility as a stressful condition doubles the risk of anxiety and depression in women (6).

Tubal ligation (TL), which is the most commonly used family planning method worldwide, is a surgical procedure for contraception in women who have completed their family or have no desire to become 
pregnant (7). Despite using TL by $19 \%$ of women aged between 15-49 years (8), sexual functioning and other aspects of QoL in women undergoing TL have not been adequately explored and available studies have found conflicting results. Several studies have reported destructive effects associated with TL on QoL and sexual function (SF)(9-13).

Childbearing as an important concept of marriage can be affected by infertility and TL. Infertile couples are those who have desire to have children of their own but are unable to do so, and therefore usually seek some type of medical infertility treatment. Nonetheless, TL is a voluntary choice for those who no longer want children and tend to limit their family size (14).

This study aimed to assess depression, anxiety, body image, self-esteem, SF, and QoL in infertile women in Iran and compare the results with fertile group (condom) and those with TL.

\section{Materials And Methods}

\section{Participants}

The study was conducted as a comparative cross-sectional approach and the sample was selected via convenience sampling method. Using the appropriate formula to calculated sample size by taking $95 \%$ confidence interval and $90 \%$ power to test. In this study, the number of participants in each group was 200 women who met the inclusion criteria.

Overall, the inclusion criteria were women who were still at their childbearing aged ( $\geq 35$ years), married, lived in the same house as her husband, and were sexually active (had sexual intercourse in the past 4 weeks). Women who were unable to conceive after 12 months of frequent coitus without contraceptives and had referred to the Royan Research Institute in Tehran (Iran) were listed as the infertile group before entering the treatment cycle. Additionally, women who had undergone tubectomy for $\geq 1$ years and women who used condoms to prevent pregnancy were included in the study when they went to healthcare centers in Tehran (Iran) for other problems. To avoid possible confounding factors, exclusion criteria included infertile couple with male infertility, gynecological disorders, suffering from chronic diseases (such as diabetes, hypertension...), not being sexually active, breastfeeding, taking any antidepressant drugs, serotonin and norepinephrine reuptake inhibitors (SNRI), or antipsychotic drugs that could have sexual side effects.

The research was conducted from May 2017 to February 2019, using a standard questionnaire that had been tested for validity and reliability and the hospital anxiety and depression scale (HADS)

Female sexual function index (FSFI), body image concern inventory (BICl), Rosenberg' Self-Esteem Scale (RSES), Short Form Health Survey (SF-12) are used to measure various aspects of women's lives. Before being asked to fill out the questionnaire, the respondents were provided with explanations and the approval letter. The study protocol was approved by the institutional review board and the Ethics Committee of the Royan Institute (IR.ACECR.ROYAN.REC.1395.97). 


\section{Questionnaires}

The participants were asked to complete several self-report questionnaires, as follows:

demographic survey that included the age of the participants, the number of their children, their

husband's age, their educational levels, occupational status, body mass index (BMI), smoking status, type of previous deliveries, and menstruation status.

\section{Anxiety and depression:}

The hospital anxiety and depression scale (HADS) objectively measure these factors and it contains a 7item depression scale and a 7-item anxiety subscale (the score range of each component is $0-3$ ). Higher scores represent higher symptom levels for both subscales and a score of 11 and above is considered a clinical disease (15). The validity and reliability of the Iranian version of HADS have been confirmed (16).

\section{Female Sexual Function:}

Female sexual function index (FSFI) investigates the quality of women's sexual life over the last month prior to the study. This questionnaire measures desire, arousal, lubrication, satisfaction, orgasm, and pain by 19 questions with a range of 0 or 1 to 5 . The domain score of 0 indicated that the women reported no sexual activity during the previous month. A total score of less than 23 denotes sexual dysfunction, and the scores less than 3.3 in the desire domain, less than 3.4 in the arousal and orgasm domain, less than 3.7 in the lubrication domain, less than 3.08 in the satisfaction and pain domain were used to classify the participants as having difficulties in that domain(17). The Persian version of FSFI has also been evaluated for both reliability and validity (18).

\section{Body Image:}

Development of the body image concern inventory $(\mathrm{BICl})$ is a measure that assesses dysmorphic concerns. The questionnaire consists of 19 questions that examine the individual's dissatisfaction and concerns about their appearance. The answer is based on the Likert scale of 1-5 and the total score range is 19-95, higher scores indicate higher levels of dissatisfaction about one's body image or appearance(19). The validity and reliability of this questionnaire has been previously assessed in Iran (20).

\section{Self-esteem:}

Rosenberg' Self-Esteem Scale (RSES) is composed of 10 questions that are scored on a 4-point Likerttype scale ranging from 1 (totally disagree) to 4 (totally agree). A higher score indicates higher selfesteem (21). Validity and reliability tests of the Iranian form have been previously performed (22).

\section{Quality of life:}


The participants were asked to fill out the Short Form Health Survey (SF-12) that measures QoL in eight areas: physical functioning, physical role, bodily pain, general health, vitality, social functioning, role emotional, and mental health by 12 items. Higher scores indicate better QoL (23). The validity and reliability of this questionnaire have been confirmed in Iran (24).

\section{Statistics}

All statistical analyses were performed by the SPSS software version 25.0 (SPSS Inc., Chicago, IL, USA). A one-sample Kolmogorov-Smirnov test was used to analyze normality for the continuous variables, which were compared using the Student's t-test and $\chi 2$-test.

A one-way ANOVA was used [with LSD posthoc test] to compare each variable between groups. Differences were considered significant at $\mathrm{P} \otimes 0.05$ for the two tails.

\section{Result}

Table 1 describes the characteristics of women in the infertile, $T L$, and condom groups. The age of the participants ranged from 20 to 43 years. As shown in Table 1, there were no significant differences $(P>0.05)$ between women age groups, the ages of the husbands, Body Mass Index, smoking status, educational level, and the type of previous deliveries in the participants' groups. However, there were statistically significant differences among the groups in characteristics of menstrual cycle length ( $28.71 \pm 2.08$ vs $27.99 \pm 5.95$ vs $29.43 \pm 6.49, P=0.02)$ and features $(P<0.001)$. The number of children in infertile, women with TL and without TL were respectively $0.83 \pm 0.38,2.35 \pm 0.56,2.29 \pm 0.71$ and there was also a statistically significant difference $(p<0.001)$.

Table 1: Comparison of demographic characteristics between infertile, TL and condom groups. 


\begin{tabular}{|c|c|c|c|c|}
\hline Variable & $\begin{array}{l}\text { Infertile group } \\
\mathrm{n}=200\end{array}$ & $\begin{array}{l}\text { TL group } \\
n=200\end{array}$ & $\begin{array}{l}\text { Condom group } \\
\mathrm{n}=200\end{array}$ & P-value \\
\hline Age of female* & $32.48 \pm 4.92$ & $33.06 \pm 3.01$ & $33.07 \pm 3.61$ & 0.25 \\
\hline Number of children* & $0.83 \pm 0.38$ & $2.35 \pm 0.56$ & $2.29 \pm 0.71$ & $<0.001$ \\
\hline Education of female** & & & & 0.08 \\
\hline Illiterate & $0(0)$ & $1(0.5)$ & $1(0.5)$ & \\
\hline Under diploma & $85(42.3)$ & $111(55.5)$ & $99(49.5)$ & \\
\hline Higher diploma & $116(57.7)$ & $88(44)$ & $100(50)$ & \\
\hline Age of husbands* & $39.06 \pm 6.27$ & $38.93 \pm 8.19$ & $38.22 \pm 4.27$ & 0.17 \\
\hline Job of female** & & & & 0.075 \\
\hline Housewife & $148(73.6)$ & $166(83)$ & $157(78.5)$ & \\
\hline Recruitment & $53(26.4)$ & $43(17)$ & $43(21.5)$ & \\
\hline BMI $(\mathrm{kg} / \mathrm{m} 2)^{*}$ & $27.02 \pm 4.39$ & $28.05 \pm 4.06$ & $27.43 \pm 4.81$ & 0.06 \\
\hline Smoking** & & & & 0.60 \\
\hline Yes & $1(0.5)$ & $1(0.5)$ & $0(0)$ & \\
\hline No & $200(99.5)$ & $199(99.5)$ & $200(100)$ & \\
\hline Menstrual cycle features** & & & & $<0.001$ \\
\hline Regular & $154(77)$ & $168(84)$ & $187(93.5)$ & \\
\hline Irregular & $46(23)$ & $32(16)$ & $13(6.5)$ & \\
\hline Menstrual cycle length (days)* & $28.71 \pm 2.08$ & $27.99 \pm 5.95$ & $29.43 \pm 6.49$ & 0.02 \\
\hline Type of delivery** & & & & 0.15 \\
\hline Cesarean section & $19(79.2)$ & $152(76)$ & $136(68)$ & \\
\hline NVD & $5(20.8)$ & $48(24)$ & $64(32)$ & \\
\hline
\end{tabular}

BMI: Body Mass Index, NVD: Natural Vaginal Delivery 


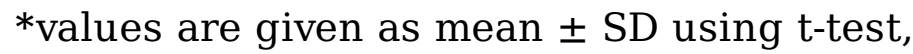

** values are given as a number (\%) using chi-squared test

\section{Sexual function status}

The evaluation of FSFI scores and comparison of sexual dysfunction are shown in Table 2. As can be seen, all mean values were lower in the TL group compared with other groups. Also, the differences in scores in the three groups were statistically significant in all domains. There was a statistically significant difference between TL, infertile, and the condom group in terms of desire, arousal, lubrication, orgasm, satisfaction, pain, and total FSFI scores. The prevalence of sexual dysfunction in the women with TL were significantly higher than the infertile and condom group $(P<0.001)$.

Table 2: Comparison of FSFI scores and dysfunction between infertile, TL and condom groups 


\begin{tabular}{|c|c|c|c|c|c|}
\hline \multirow[t]{3}{*}{ Variable } & $\begin{array}{l}\text { Infertile group } \\
\text { (I) }\end{array}$ & $\begin{array}{l}\text { TL group (T) } \\
\mathrm{N}=200\end{array}$ & $\begin{array}{l}\text { Condom group } \\
\text { (C) }\end{array}$ & P-value* & $\begin{array}{l}\text { Pair wise } \\
\text { comparison }\end{array}$ \\
\hline & $N=200$ & Mean \pm SD & $\mathrm{N}=200$ & & P-value** \\
\hline & Mean $\pm S D$ & & Mean $\pm S D$ & & \\
\hline \multirow[t]{3}{*}{ Desire } & $3.79 \pm 0.83$ & $3.07 \pm 0.84$ & $3.99 \pm 0.75$ & $<0.001$ & I and C: 0.012 \\
\hline & & & & & I and $\mathrm{T}:<0.001$ \\
\hline & & & & & T and C: $\square 0.001$ \\
\hline \multirow[t]{3}{*}{ Arousal } & $3.99 \pm 1.08$ & $3.28 \pm 1.01$ & $4.20 \pm 0.82$ & $<0.001$ & I and C: 0.003 \\
\hline & & & & & $\mathrm{I}$ and $\mathrm{T}:<0.001$ \\
\hline & & & & & T and C: 0.001 \\
\hline \multirow[t]{3}{*}{ Lubrication } & $4.61 \pm 1.20$ & $3.82 \pm 1.19$ & $4.91 \pm 0.76$ & $<0.001$ & I and C: 0.005 \\
\hline & & & & & I and $\mathrm{T}:<0.001$ \\
\hline & & & & & T and C: 0.001 \\
\hline \multirow[t]{4}{*}{ Orgasm } & $3.06 \pm 0.91$ & $3.81 \pm 1.30$ & $4.89 \pm 0.77$ & $<0.001$ & I and C: $\square 0.001$ \\
\hline & & & & & I and $\mathrm{T}:<0.001$ \\
\hline & & & & & and \\
\hline & & & & & $C:<0.001$ \\
\hline \multirow[t]{4}{*}{ Satisfaction } & $4.74 \pm 1.12$ & $4.19 \pm 1.15$ & $5.14 \pm 0.88$ & $<0.001$ & I and C: $\square 0.001$ \\
\hline & & & & & I and $\mathrm{T}:<0.001$ \\
\hline & & & & & and \\
\hline & & & & & $C:<0.001$ \\
\hline \multirow[t]{4}{*}{ The pain } & $4.67 \pm 1.28$ & $4.18 \pm 1.44$ & $4.87 \pm 0.96$ & $<0.001$ & I and C: 0.102 \\
\hline & & & & & I and $\mathrm{T}:<0.001$ \\
\hline & & & & & and \\
\hline & & & & & $C:<0.001$ \\
\hline
\end{tabular}




\section{Dysfunction***}

Yes

No

$148(73.6)$

$177(88.5)$

$53(26.4)$
$115(57.5)$

$<0.001$

$85(42.5)$

FSFI: Female Sexual Function Index, TL: tubal ligation. ANOVA: analysis of variance

* One-way ANOVA

** One-way ANOVA followed by LSD post hoc test

***values are given as a number (\%) using chi-squared test

\section{Quality of life status}

Table 3 compares SF-12 scores between the infertile, $T L$, and condom groups. The mean total scores of SF-12 was significantly lower in the TL group compared with the other groups $(76.59 \pm 13.14$ vs $68.49 \pm 14.47$ vs $78.87 \pm 12.62 \mathrm{P} \otimes 0.001)$.

Table 3: Sums scores and total scores for the domain subgroups of QoL, HADS, and body image between infertile, TL, and condom group 


\begin{tabular}{|c|c|c|c|c|c|}
\hline \multirow[t]{4}{*}{ Variable } & Infertile & group & Condom & $\mathrm{P}-$ & Pair \\
\hline & group (I) & $(\mathrm{T})$ & group (C) & value* & comparison \\
\hline & $\mathrm{n}=200$ & $n=200$ & $\mathrm{n}=200$ & & P-value ${ }^{* *}$ \\
\hline & Mean $\pm S D$ & Mean $\pm S D$ & Mean $\pm \mathrm{SD}$ & & \\
\hline
\end{tabular}

SF-12

Sum score $78.96 \pm 14.10 \quad 67.88 \pm 15.87 \quad 79.52 \pm 14.25<0.001 \quad$ I and C: 0.70 physical

I and

$\begin{array}{ll}\text { Components } & \mathrm{T}:<0.001\end{array}$

(PCS-12)

$\mathrm{T}$

and

C: $\square 0.001$

Sum 74.22 $\pm 15.23 \quad 69.10 \pm 16.0278 .23 \pm 13.34<0.001 \quad I \quad$ and $\quad$ C:

score mental

0.007

Components

I and T:

(MCS-12)

$<0.001$

$\mathrm{T}$ and

C:

$<0.001$

Total score

$76.59 \pm 13.14$

$68.49+14.47$

$78.87 \pm 12.62$

(

$6.59 \pm 13.14 \quad 68$.

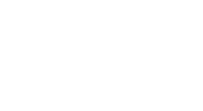

I and $\mathrm{T}:$
$<0.001$
$\mathrm{~T} \quad$ and
$\mathrm{C}:<0.001$

\section{HADS}

Depression

$5.29 \pm 3.22 \quad 6.65 \pm 3.57$

$4.42 \pm 3.22$

$<0.001$

I and C: 0.09

I and $\mathrm{T}$ :

$<0.001$ 
$\mathrm{C}:<0.001$

Anxiety

$7.66 \pm 3.05$

$10.05 \pm 3.93$

$6.84 \pm 3.27$

$<0.001$

and

C:

0.005

I and T:

$<0.001$

$\mathrm{T}$ and $\mathrm{C}$

$\square 0.001$

Total scores

$12.88 \pm 4.93 \quad 16.55 \pm 6.49$

$11.23 \pm 5.97$

$<0.001$

0.017

I and $\mathrm{T}$ :

$<0.001$

$\mathrm{T}$

and

$\mathrm{C}:<0.001$

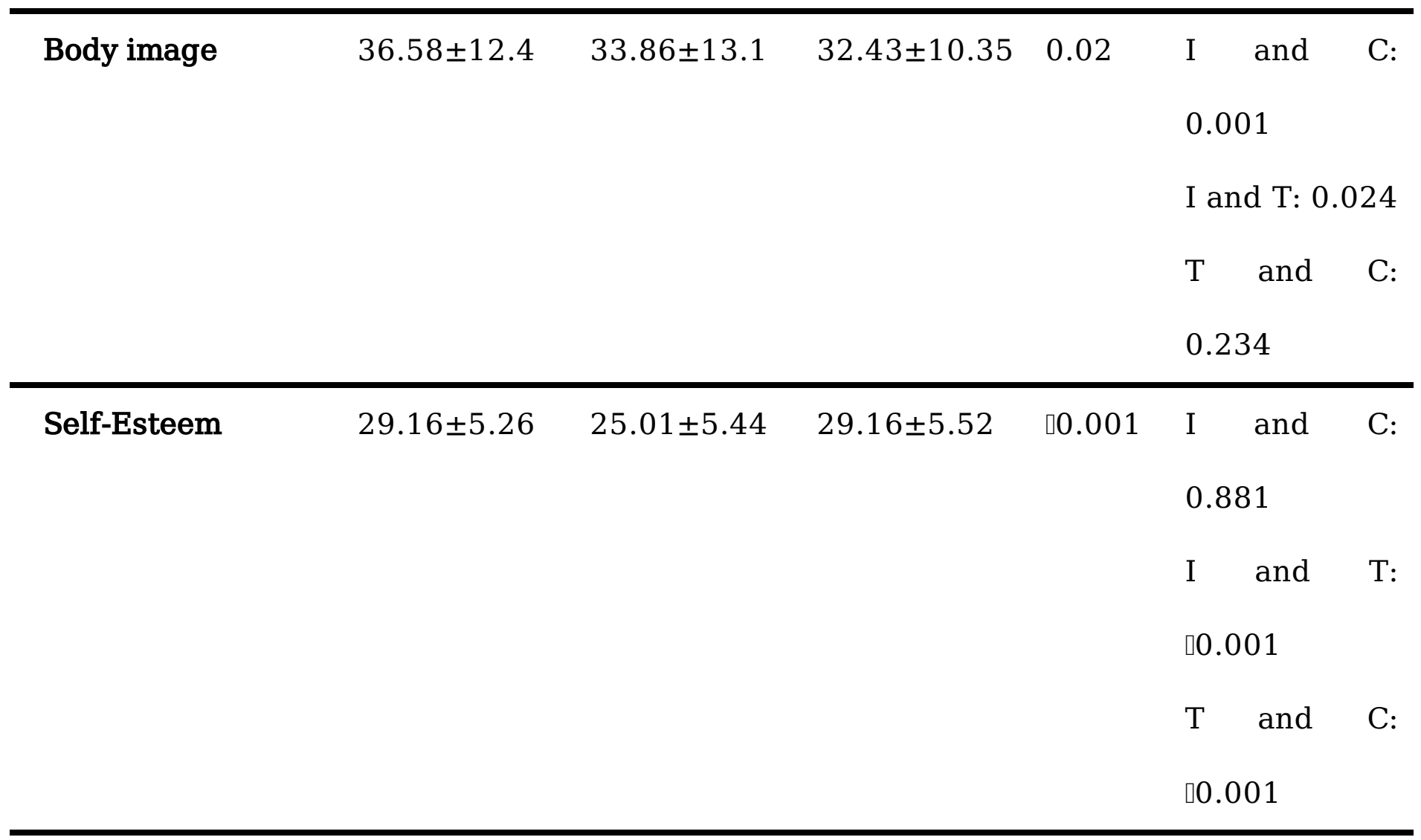


QoL: Quality of Life, SF-12: Short Form-12, HADS: Hospital Anxiety and Depression Scale, TL: Tubal Ligation

* One-way ANOVA

** One-way ANOVA followed by LSD post hoc test.

From the information summarized in Table 3 we can see that the TL group shows significantly less physical, psychological, and total scores of QoL than the other two groups (P凶0.001). There was no significant difference between infertile and condom groups in physical and total scores of QoL $(P>0.05)$.

\section{Depression and anxiety}

As shown in Table 3, there were statistically significant differences between the mean scores of depression, anxiety, and total HADS in infertile, TL, and condom groups ( $12.88 \pm 4.93$ vs $16.55 \pm 6.49$ vs

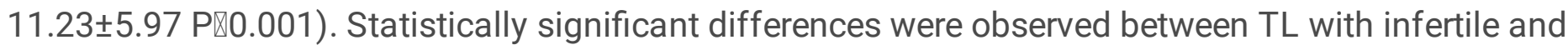
condom groups in depression, anxiety, and total scores of HADS. However, there was no statistically significant difference between infertile and condom group in terms of depression $(P>0.05)$.

\section{Body image and Self-esteem}

It can be seen from the data in Table 3 that the body image score difference was statistically significant among $T L$, infertile and condom groups $(P \Downarrow 0.05)$ and infertile women have a higher score $(36.58 \pm 12.4 \mathrm{vs}$ $33.86 \pm 13.1$ vs $32.43 \pm 10.35, P=0.02)$. Self-esteem was statistically lower in infertile women $(29.16 \pm 5.26$ vs $25.01 \pm 5.44$ vs $29.16 \pm 5.52, P \llbracket 0.001)$.

\section{Discussion}

According to the increasing infertility rate in Iran, the main goal of the current study was to assess SF, QoL, depression, anxiety, self-esteem, and body image among infertile women. Also, due to the soaring use of TL by Iranian women in recent years, it is important to evaluate potential complications associated with this method. Recognition and diagnosis of sexual dysfunction as well as the impairment of QoL, indicate a need for sufficient education and comprehensive consultation by the healthcare system prior to the procedure.

\section{Anxiety and Depression}

Although TL is the most commonly used form of contraceptive method worldwide, the long term psychological effects are still obscure. Contrary to expectations, the result of this study showed that anxiety and depression symptoms in women who had undergone TL surgery would be higher than that in infertile women.

Many evidences indicated that depression and anxiety are more prevalent among women who had infertility compared to non-infertile women. Almost half of the infertile subjects had moderate to severe symptoms of depression and the level of anxiety was increased by infertility (25). Anxiety and 
depression caused by infertility may be due to various factors such as uncertainty about the cause of infertility, duration of treatment, and unspecified treatment, as well as financial and social pressures. Since cyclical hormonal changes are related to anxiety and depression, these observations may be due to undergoing TL (26). The results of a study by Lin et al. (27) indicated sterilization as a risk factor for depression and anxiety; the risk of depression and anxiety following TL was reported to be 2.34 and 2.88 times greater than before TL, respectively. A similar study has been conducted on one hundred sixty-two women who had experienced TL, reported increased Beck depression inventory scores after TL and were suffering from regret after sterilization (26). Complications by reason of TL cause regret (14), in addition to in traditional societies such as Iran, the picture of a woman is directly related to her fertility and motherhood ability and cause them to feel perfect and valuable. Although TL is a voluntary procedure, cultural factors that are rooted in one's unconscious cause conveys them feelings of inadequacy and unattractive that can manifest as sexual dysfunction and regret (9). It has been shown in several studies that anxiety and depression are more common in women regretting sterilization in comparison to the control group $(14,26)$.

Depression can be influenced by many factors including a feeling of guilt after an irreversible surgery, negative perceptions of others, a change in husband's intuition, cultural conditions, and religion status, or pre-existing emotional disorders. Women should be given time to consider their decision on fertility state and be provided with psychological support during this time.

\section{Body image and Self-esteem}

In the results of our study, infertile women had lower body image in comparison with TL and condom group. These results confirm the association between body image and infertility. In addition to physical appearance, a person's body image also reflects physical wellbeing and biological status (health/illness) (28). Previous studies have shown that self-concept, identity development, anxiety, and depression are factors closely related to a woman's body image that might be affected by infertility (29). A strong relationship between infertility and depression has been reported in the literature (28), so depression in different stages of life may lead to a distorted body image (29). Based on our findings, self-esteem scores were lower in TL women compared to the other two groups. In many studies, however, the authors have indicated that there was no link between TL and self-esteem $(11,30)$.

This observation may support the hypothesis that although individuals continuously think about their appearances, after a physical disease state like infertility, they become more aware and alert of their bodies and are more mentally concerned (31).

In sum, very little research has been done on the relationship between undergoing TL and body image. $\mathrm{Li}$ et al. (11) and Raine et al. (30) showed that TL had no effect on body image. Further work is required to establish the validity of this result and different factors affecting the body image.

\section{Sexual function status}


In the present study, the prevalence of FSD in the TL women was around $50 \%$ in comparison to $27.4 \%$ in infertile group and $8.5 \%$ in condom groups. Therefore, results from many studies have confirmed that women with infertility had an increase in sexual dysfunction(32-34). Omani-Samani (35) carried out a meta-analysis study to estimate the prevalence of FSD among infertile Iranian women. Their result suggested that more than $64 \%$ of infertile Iranian women reported sexual dysfunction. It is well-known that the risk of FSD in infertile women is higher because the tendency of having sexual intercourse is strongly affected by pregnancy. Among infertile couples, sex is defined as a clinical tool, which should happen on certain days of the month, instead of an act of love (36). It has, on the other hand, been suggested that in some cases infertility might be a result of sexual dysfunction (37). The results of studies that have assessed the effects of TL on SF are controversial. Surprisingly, Smith et al. (12) and Li et al. (11) observed positive effects of sterilization on SF, which may be the result of reduced fear of getting pregnant. In contrast, Kunkeri et al. (38) and Jahanian Sadatmahalleh et al. (9) reported the higher FSD in women undergoing TL compared with their control group.

According to the proven effects of infertility on the quality of SF (32), this finding was unexpected. But a possible explanation for this might be "post tubal sterilization syndrome" that causes a decrease of libido, menstruation disorders, pelvis pain, dyspareunia, and depression (39).

SF is complex and involves interactions of many factors, including emotional connection, body image, and other elements such as cultural differences, ethnicity, misinterpretation of religious codes, personal belief regarding her role as a woman, and social pressures $(40,41)$. Overall, it seems that having a discussion about sexual matters is centuries-long taboo, especially speaking about them with others, including the spouse and their doctor (38).

Regarding the importance of SF in life, marital relationship and the significant association between poststerilization regret and FSD in women with TL (44), Jahanian Sadatmahalleh et. al. reported the prevalence of FSD in the TL group was $63.4 \%$ in comparison with $40.8 \%$ in the control group (14). Being completely informed about TL procedure and its complications, and having access to other contraceptive options prior to the operation may be helpful in avoiding future dissatisfaction.

\section{Quality of life status}

We found that QoL in women with TL (both physical and mental demands) was significantly lower than other groups.

Several reports have shown that infertility is associated with decreased physical and mental health(45, 46). The result of a qualitative study which conducted on 16 people who received successful infertility treatment supported evidence from previous observations. The effects of the stage of infertility process, gender, and the quality of the relationship on marital relationship and QoL have been indicated; infertility causes many problems such as dissatisfaction, stress, sadness, insomnia, increased/decreased appetite, increased smoking, social pressure, exposure to questions of curiosity about having children, avoiding from being where children are, losing the privacy of sex life, having sex in some planned days just for 
reproduction, interruption in work life and the high cost of treatment. Therefore, it is probable that the scores of QoL have decreased in infertile women (47).

Numerous studies which have evaluated the impact of TL on women's QoL, have observed conflicting results. For instance Pauls R. (13) conducted a study to summarize recent literature on SF following benign gynecological surgeries, including tubal ligation. They concluded that the QoL and SF in the majority of the cases benefit from surgical interventions. However, Li et. al. (11) did not find such associations. Thus far, several studies have demonstrated that TL has a negative impact on QoL $(9,10)$. In a similar study that was conducted by Monga et. al. (48) 18 infertile couples and 12 couples seeking elective sterilization were interviewed. The total marital adjustment and quality of well-being's score of the women who seek infertility treatment were lower than in women with elective sterilization.

One possible interpretation for our result could be the occurrence of menstrual abnormalities after TL (49). The spectrum of menstrual disorders can be wide and includes more frequent menstrual periods, irregular menstrual cycles, menorrhagia, metrorhagia, spotting, dysmenorrhea, and oligo menorrhea (50). Given the importance of the menstrual pattern in Iranian women's lives, any menstrual disorder can have a devastating effect on personal life and religious duties. Therefore, TL is a decision that can later cause women to feel guilty.

Discrepancies in QoL and SF conditions after TL surgery can be the result of different definition of TL in various cultures. In societies with traditional cultures, such as Iran, self-satisfaction, value, and the image that a woman holds of herself is closely reliant on their motherhood ability. Although TL seems to be a voluntary choice at first, it might induce a feeling of guilt in some women. Therefore, sexual dysfunction, a decrease in QoL, and regret for having done this operation might happen after a while (9). In sum, due to the growing demand for TL surgery as a means of contraception, the number of women who regret choosing this method as a permanent approach have been increased (51). Regression is associated with sadness, pain, harm, affliction, anxiety, and displeasure, all of which affect the QoL (52).

Despite the fact that, multiple psychological and physical factors such as FSD affect a woman's QoL (53), many physicians refuse to look for details about SF and women often do not perceive it as a disorder and never discuss this with their doctors, so many women have suffered in silence for years (38). Women should view SF as a biological and enjoyable need and be free to talk about their sexual problems, and this cannot be achieved except through education and counseling (38).

Despite the well-known adverse effects of infertility on women's health, the results of this study imply the relationship between TL and the poor quality of various aspects of a woman's life. Based on our knowledge, this study has compared infertile and TL women for the first time and has provided a great value for fertility and childbearing in societies like Iran. This study distinguishes the importance of making the right decisions about anything that may affect fertility more than previous studies. Nonetheless, this study was limited by the absence of sufficient information about the history of QoL, FSD, and the mental health of individuals before TL surgery. A larger sample size would be helpful to determine more significant results. 


\section{Conclusion}

This study has identified SF, QoL, psychological health in women undergoing TL and has shown that they were more disrupted compared with infertile and condom groups. To our knowledge it is for the first time that such a study has been conducted in Iran. These findings have significant implications and suggest a need for investigating the side effects of TL more carefully, as TL may not be a safe method for contraception. This study has provided profound insight into the importance of a comprehensive consultation before TL.

\section{List Of Abbreviations}

FSFI: Female Sexual Function Index

SF: Sexual Function

FSD: Female Sexual Dysfunction

HADS: Hospital Anxiety and Depression Scale

QoL: Quality of Life

BMl: Body Mass Index

TL: Tubal Ligation

\section{Declarations}

\section{Ethics approval and consent to participate}

The study protocol was approved by the Ethics Committee of the Royan Institute (code: IR.ACECR.ROYAN.REC.1395.97). All procedures were in accordance with the ethical standards of the Regional Research Committee and with the Declaration of Helsinki 1964 and its later amendments. After explaining the study's purposes, a written consent and a verbal assent were collected from all participants, and the subjects were informed that their participation was voluntary, confidential, and anonymous and that they had the right to withdraw from the research at any time.

\section{Consent for publication}

Not applicable.

\section{Availability of data and materials}

The data sets used and analyzed for the current study are available upon reasonable request. 
The authors declare no conflicts of interest.

\section{Funding}

None.

\section{Authors' contributions}

Sh.JS and M.A contributed to the conception and design of the study; M.A and Sh.JS did the literature search; N.M and Sh.JS performed the statistical analysis; M.A, N.M, Sh.JS, N.J and S.Y wrote the first draft of the manuscript. All authors contributed to manuscript revision, read, and approved the submitted version.

\section{Acknowledgments}

This study was carried out with the kind collaboration of the participants. We would also like to appreciate the staff of a Royan Institute and the participating health care centers for their valuable contributions.

\section{References}

1. Whiteford LM, Gonzalez L. Stigma: the hidden burden of infertility. Social Science and Medicine. 1995;40(1):27-36.

2. Benyamini Y, Gozlan M, Kokia E. Variability in the difficulties experienced by women undergoing infertility treatments. Fertility and Sterility. 2005;83(2):275-83.

3. Warner L, Jamieson DJ, Barfield WD. CDC releases a national public health action plan for the detection, prevention, and management of infertility. Journal Of Women's Health. 2015;24(7):548-9.

4. Huppelschoten AG, Van Dongen A, Verhaak C, Smeenk J, Kremer J, Nelen W. Differences in quality of life and emotional status between infertile women and their partners. Human Reproduction. 2013;28(8):2168-76.

5. Bakhtiyar K, Beiranvand R, Ardalan A, Changaee F, Almasian M, Badrizadeh A, et al. An investigation of the effects of infertility on Women's quality of life: a case-control study. BMC women's health. 2019;19(1):114.

6. Baldur-Felskov B, Kjaer S, Albieri V, Steding-Jessen M, Kjaer T, Johansen C, et al. Psychiatric disorders in women with fertility problems: results from a large Danish register-based cohort study. Human reproduction. 2013;28(3):683-90.

7. WHO. Family planning/Contraception 22 June 2020 [Available from: https://www.who.int/newsroom/fact-sheets/detail/family-planning-contraception. 
8. Papaharitou S, Nakopoulou E, Kirana P, Iraklidou M, Athanasiadis L, Hatzichristou D. Women's sexual concerns: Data analysis from a help-line. The Journal of Sexual Medicine. 2005;2(5):652-7.

9. Sadatmahalleh SJ, Ziaei S, Kazemnejad A, Mohamadi E. Evaluation of sexual function and quality of life in Iranian women with tubal ligation: a historical cohort study. International journal of impotence research. 2015;27(5):173-7.

10. Gulum M, Yeni E, Sahin M, Savas M, Ciftci H. Sexual functions and quality of life in women with tubal sterilization. International journal of impotence research. 2010;22(4):267-71.

11. Li RH, Lo SS, Teh DK, Tong N-C, Tsui MH, Cheung K-B, et al. Impact of common contraceptive methods on quality of life and sexual function in Hong Kong Chinese women. Contraception. 2004;70(6):474-82.

12. Smith A, Lyons A, Ferris Ja, Richters J, Pitts M, Shelley J. Are sexual problems more common in women who have had a tubal ligation? A population-based study of Australian women. BJOG: An International Journal of Obstetrics \& Gynaecology. 2010;117(4):463-8.

13. Pauls R. Impact of gynecological surgery on female sexual function. International journal of impotence research. 2010;22(2):105-14.

14. Sadatmahalleh SJ, Ziaei S, Kazemnejad A, Mohamadi E. Evaluation of influencing factors on tubal sterilization regret: a cross-sectional study. International journal of fertility \& sterility. 2018;12(3):200.

15. Snaith RP. The hospital anxiety and depression scale. Health and quality of life outcomes. 2003;1(1):1-4.

16. Montazeri A, Vahdaninia M, Ebrahimi M, Jarvandi S. The Hospital Anxiety and Depression Scale (HADS): translation and validation study of the Iranian version. Health and quality of life outcomes. 2003;1(1):14.

17. Rosen CB, J. Heiman, S. Leiblum, C. Meston, R. Shabsigh, D. Ferguson, R. D'Agostino, R. The Female Sexual Function Index (FSFI): a multidimensional self-report instrument for the assessment of female sexual function. Journal of sex \& marital therapy. 2000;26(2):191-208.

18. Heydari M, Faghihzadeh S. The female sexual function index (FSFI): validation of the Iranian version. Payesh (Health Monitor). 2008;7(3):0-.

19. Littleton HL, Axsom D, Pury CL. Development of the body image concern inventory. Behaviour Research and therapy. 2005;43(2):229-41.

20. MOHAMMADI NE, Sajadinezhad M. The evaluation of psychometric properties of body image concern inventory and examination of a model about the relationship between body mass index, body image dissatisfaction and self-esteem in adolescent girls. 2007.

21. Rosenberg M. Rosenberg self-esteem scale (RSE). Acceptance and commitment therapy Measures package. 1965;61(52):18.

22. Shapurian R, Hojat M, Nayerahmadi H. Psychometric characteristics and dimensionality of a Persian version of Rosenberg Self-esteem Scale. Perceptual and Motor Skills. 1987;65(1):27-34. 
23. Ware Jr JE, Kosinski M, Keller SD. A 12-Item Short-Form Health Survey: construction of scales and preliminary tests of reliability and validity. Medical care. 1996:220-33.

24. Montazeri A, Vahdaninia M, Mousavi SJ, Omidvari S. The Iranian version of 12-item Short Form Health Survey (SF-12): factor structure, internal consistency and construct validity. BMC public health. 2009;9(1):341.

25. Lakatos E, Szigeti JF, Ujma PP, Sexty R, Balog P. Anxiety and depression among infertile women: a cross-sectional survey from Hungary. BMC women's health. 2017;17(1):48.

26. Kelekçi S, Erdemoglu E, Kutluk S, Yılmaz B, Savan K. Risk factors for tubal ligation: regret and psychological effects impact of Beck Depression Inventory. Contraception. 2005;71(6):417-20.

27. Lin L, Shi-Zhong W, Changmin Z, Qifu F, Keqiang L, Goliang S. Psychological long-term effects of sterilization on anxiety and depression. Contraception. 1996;54(6):345-57.

28. Akhondi MM, Dadkhah A, Bagherpour A, Ardakani ZB, Kamali K, Binaafar S, et al. Study of body image in fertile and infertile men. Journal of Reproduction \& Infertility. 2011;12(4):295.

29. Gibson DM, Myers JE. Gender and infertility: A relational approach to counseling women. Journal of Counseling \& Development. 2000;78(4):400-10.

30. Raine T, Minnis AM, Padian NS. Determinants of contraceptive method among young women at risk for unintended pregnancy and sexually transmitted infections. Contraception. 2003;68(1):19-25.

31. Wallach EE, Mahlstedt PP. The psychological component of infertility. Fertility and sterility. 1985;43(3):335-46.

32. de Mendonca CR, Arruda JT, Noll M, Paulo MdO, do Amaral WN. Sexual dysfunction in infertile women: A systematic review and meta-analysis. European Journal of Obstetrics \& Gynecology and Reproductive Biology. 2017;215:153-63.

33. Ozturk S, Sut HK, Kucuk L. Examination of sexual functions and depressive symptoms among infertile and fertile women. Pakistan journal of medical sciences. 2019;35(5):1355.

34. Gabr AA, Omran EF, Abdallah AA, Kotb MM, Farid EZ, Dieb AS, et al. Prevalence of sexual dysfunction in infertile versus fertile couples. European Journal of Obstetrics \& Gynecology and Reproductive Biology. 2017;217:38-43.

35. Omani-Samani R, Maroufizadeh S, Almasi-Hashiani A, Amini P. Prevalence of depression and its determinant factors among infertile patients in Iran based on the PHQ-9. Middle East Fertility Society Journal. 2018;23(4):460-3.

36. Zare Z, Amirian M, Golmakani N, Mazlom R, Ahangar ML. Sexual dysfunction in infertile women. International Journal of Reproductive BioMedicine. 2016;14(2):89.

37. Bakhtiari A, Basirat Z, Nasiri-Amiri F. Sexual dysfunction in women undergoing fertility treatment in Iran: prevalence and associated risk factors. Journal of reproduction \& infertility. 2016;17(1):26.

38. Kunkeri SP, Rao TS, Andrade C. Study of sexual functioning and disorder in women before and after tubal sterilization (tubectomy). Indian Journal of Psychiatry. 2017;59(1):63.

39. Cattanach J. Oestrogen deficiency after tubal ligation. The Lancet. 1985;325(8433):847-9. 
40. González M, Viáfara G, Caba F, Molina T, Ortiz C. Libido and orgasm in middle-aged woman. Maturitas. 2006;53(1):1-10.

41. Imeni B, Karampourian A, editors. Investigating the effect of sexual satisfaction on family stability. Proceedings of the 2nd National Congress on Family and Sexual Problems; 2005.

42. Opiyo-Omolo B. The continuum complete international encyclopedia of sexuality: A\&C Black; 2004.

43. Sayasneh A, Pandeva I. Postpartum Sexual Dysfunction: A literature review of risk factors and role of mode of delivery. Bjmp. 2010;3(2):316.

44. Warehime MN, Bass L, Pedulla D. Effects of tubal ligation among American women. The Journal of reproductive medicine. 2007;52(4):263-72.

45. Namdar A, Naghizadeh MM, Zamani M, Yaghmaei F, Sameni MH. Quality of life and general health of infertile women. Health and Quality of life Outcomes. 2017;15(1):139.

46. Boulet SL, Smith RA, Crawford S, Kissin DM, Warner L. Health-Related Quality of Life for Women Ever Experiencing Infertility or Difficulty Staying Pregnant. Maternal and Child Health Journal. 2017;21(10):1918-26.

47. Onat G, Beji NK. Marital relationship and quality of life among couples with infertility. Sexuality and Disability. 2012;30(1):39-52.

48. Monga M, Alexandrescu B, Katz SE, Stein M, Ganiats T. Impact of infertility on quality of life, marital adjustment, and sexual function. Urology. 2004;63(1):126-30.

49. Williams EL, Jones HE, Merrill RE. The subsequent course of patients sterilized by tubal ligation: a consideration of hysterectomy for sterilization. American journal of obstetrics and gynecology. 1951;61(2):423-6.

50. AtashKhoii S. The risk of menstrual abnormalities after tubal sterilization: a case control study. BMC women's health. 2005;5(1):5.

51. Pollack A, Bulletins-Gynecology ACoP. ACOG practice bulletin. Clinical management guidelines for obstetrician-gynecologists. Number 46, September 2003. (Replaces technical bulletin number 222, April 1996). Obstetrics and gynecology. 2003;102(3):647-58.

52. Ludermir AB, Machado KMdM, Costa AMd, Alves SV, Araújo TVBd. Tubal ligation regret and related risk factors: findings from a case-control study in Pernambuco State, Brazil. Cadernos de Saúde Pública. 2009;25:1361-8.

53. Oksuz E, Malhan S. Prevalence and risk factors for female sexual dysfunction in Turkish women. The Journal of urology. 2006;175(2):654-8. 